\title{
One universe, endless cycles
}

\author{
Edwin Zong \\ Oasis Medical Group, Bakersfield, CA 93305, USA
}

Email address:

R4gomed@yahoo.com

\section{To cite this article:}

Edwin Zong. One Universe, Endless Cycles. Science Research. Vol. 2, No. 5, 2014, pp. 105-110. doi: 10.11648/j.sr.20140205.15

\begin{abstract}
The finding of cosmic cold spots now reveal real possibilities of existing other universe. Many researchers are now aware of our universe might be the one of billions-perhaps an infinite number of universes(1). However, the introduction of concept of multiverse creates confusion, because it alters the definition of universe established in scientific world.. In this paper, a possible cause is discussed in physics. The theories of multiverse indirectly recognize possibilities of multiple big bangs' existence, where each big bang generates its respective family of galaxies and occupies it's associated space. Based on recent telescope observation, our universe is cooling down while its expanding is sped up (2) The energy in the universe is constant, when the background temperature drops, the heat energy transforms into a different form of energy that is momentum which fueling passing galaxy with kinetic energy(3). In this paper, author will clarify some of paradox phenomenon that puzzles physics theorists who follow classic physics law. The introduction of new physics law-e.g. quantum law is neither necessary nor supported. The big bang explosion is triggered by a certain level (quantifiable) of mass/energy. It cannot require an infinite level of mass/energy. To explain it in a more organized way without defying universe definition, A new terminology - "Zolaxy" must be introduced for each part of the universal system, (3) which is created by a single mother blast.
\end{abstract}

Keywords: Big Bang, Origin of Universe, Black Matters, Particles, Time, Odds, Single Bang, Numerous Bangs, Quantum

\section{Introduction}

Few will argue today that our universe space is infinite in size, and it does have a background temperature, which means energy, according to Einstein's equation where $\mathrm{E}$ is energy, $\mathrm{m}$ is mass, and $\mathrm{c}$ is the speed of light in a vacuum (true void of any known or unknown particles/energy). When $\mathrm{E}=0, \mathrm{M}=0$. Energy doesn't exist without matter. Based on observation, the universe is cooling but the peripheral galaxies are speeding up. where are those kinetic energy from? It has puzzled many scientists. Some scientists may have doubts about big bang because of it.. During the life cycle of universe, was it just a single big bang that created this current universe some 14 billion years ago? The equally confusion question may also exist- is the big bang triggered by a limited amount of mass/energy reached within or it requires infinite amount of mass/energy? Based on study of sub-atom particles, some extreme physicists now promote a concept of quantum mechanics; they believe "it is possible that something can come out of nothing". This concept has dramatically deviated from classic physics. Are they correct about the nature? Or they are just romantic physics fantasists? Lastly, do we really need to reject the definition of universe, which is a simple noun well established and accepted by humanity? Can Multiverse replace universe become a new noun simply take over all definition Universe once own? Furthermore, should we change the definition of universe to fit into a smaller cosmic system? The primary objective of this study is to explain some of paradox universe phenomenon in the discipline of classic physics and philosophy, to clarify potential chaos in our cosmic naming system.

\section{Integration of Theoretical Physics, Math and Philosophy}

The universe is infinite by definition, not by scientific observation. We invent the word "universe" with a definition that covers all space and matter. If something else exists outside of the universe, whether it is space or matter, then we need to reject the definition of universe and coin a new word which will include outside space/matter. "The universe is finite" cannot be accepted, because it violates logic. It is a logical consensus that we accept this definition of "universe," which covers everything, both known and unknown. Thus, 
Universe is well known and well established cosmic word, no reason to change it or replace it with another name which simply takes over its entire definition. The creation of word multiverse is identical with current word of universe by definition, but down size universe to smaller version fit into smaller version of cosmic system. This change apparently will create confusion among world population with no scientific advantage. Since multiverse theory indirectly admits numerous big bangs which own it's respective universe, the logical way to build a name system is to accept current UNIVERSE definition, because it doesn't conflict our latest observations. Under UNIVERSE umbra, it is logical to coin a new cosmic family name-Zolaxy which collects all cosmic matters coming from one single origin- a related big bang. Without changing anything, now we can continue say our universe space is infinite in size, and it does have a background temperature, which means energy, according to Einstein's equation Energy doesn't exist without matter. Therefore, the matter in universe is infinite as well. Otherwise, you cannot explain how limited matter could fill an unlimited space and give a background temperature of 2.7 $\mathrm{K}$. In other words, temperature is another form of matter. Look at Einstein's equation again. $\mathrm{E}=\mathrm{MC}^{2}$. If temperature exists in every part of the universe, then energy is infinite by definition as well, simply because the space of the universe is infinite by definition. Limited energy/matter cannot disperse in an infinite space. The matter in the universe therefore is defined as infinite by logical reasoning. Those matters cannot be increased or decreased, which gives the fact that universal matter is constant, and universal energy is constant correspondingly. Science can never measure infinity because of its inherent deficiency: It is the fact that the physical size of any manmade object/instrument cannot reach infinite. That's where logic/human philosophy is called in to help science. The primitive matter/particles - e.g. cosmic strings, or any particles - existing in the background of the universe are not what we have in our periodic table of elements, which ranges from the lightest element, hydrogen, to super heavy ones, e.g. plutonium. Elements in our periodic table can be changed by adding or removing protons (a process also known as fusion or fission, in nuclear weapons terminology) from the nucleus, along with some electrons.

\subsection{Sub-Atom Particles}

At sub-atom level, whether it is called god particle or ghost particle, it appears manifest yin and yang character with inter-transform swiftly under certain condition. The primitive matter/particles are building blocks for nucleus in atoms; after a big bang, some of those sub-nucleus particles remain unengaged in the formation of atoms, they remain free and they may contribute to cosmic strings/ray or wave or black hole. The so-call dark matters are no magic but same as visible matters per se. because all those matters may manifest its visibility character which theoretically can be detected by tele/micro-scope and carry energy which theoretically can be detected by energy detectors. Atoms from our periodic table are the building blocks for our molecules, and later on, some of those molecules may be integrated into nonorganic chemical compounds, as well as engaged into their sophisticated sisters-organic compounds. Organic compounds later engage into organic lives. To prove where those living beings or organic materials from, First, all living beings can be resolved back to their organic building blocks through a process of fermentation or decaying of body. Secondly, those sophisticated sister compounds can be further broken down to inorganic materials or molecules by burning. Those basic molecules can be even further broken down to their most primitive particles. For Example, leave a steak in the center of active nuclear reactor; you will know what that steak turns to be-atoms and particles. However, we do not have the technology to restore those particles back to a steak. Thus, those primitive particles are generated by big bang/mega nuclear or sub nuclear alike explosions. It is a round trip. The big bang explosion is most likely not equal to the nuclear explosions known to humans today. It is more likely a gigantic explosion/big bang of measurable amount of primitive particles that reach critical level first- a direct result of mass merging e.g. gathering of enormous numbers of dying galaxies. At micro level, the impact of background energy particle on experiment target particles is much greater vs. study on target large mass for experiment purpose (neglectable impacts to its least). The absolute void space must be $0 \mathrm{k}$ on the scale of absolute temperature. Without such strict environment, none of studies on target particles is valid. Thus, the irrational conclusions from those invalid studies (e.g. quantum law/mechanics) are nothing but human fantasy. The black hole/black matter is a collection of primitive particles, which is resulted from merging/collapsing/condensing of colossal masses. The black hole doesn't take too much space, because the space and distance existing in matters is dramatically eliminated. Black matter is just primitive particles, acting differently from its super-structure brothers e.g. atoms or molecules. However, the black matters or any particles still follow the law of Einstein Equation, because it carries energy even it is invisible in today's technology. In reality, the stealth fighter jet may have a way to avoid detection from radar, but it cannot hide itself from an energy detector since it is mass/energy per se. Similarly, black hole may have a way to avoid being detected from regular telescope, but it cannot hide itself from an energy detector either. The separation of energy from mass or vice versa will violate the Einstein Equation.

\subsection{Basic Energy Form in Universe}

In universe, there are two basic forms of energy-Kinetic and Potential (any existing energy falls into one of these two). The kinetic energy (e.g. Momentum) prevents stars and planets merge into each other. On the other hand, the potential energy (e.g. gravitational potential) attracts all planets merge to each other. When planets, stars, galaxies are at their young/mature age, they have enough kinetic energy retrieved from a mother bang keep them away from each other. When time passes, their kinetic energy decline due to 
traveling a long distance in space after bang, more and more aging stars/planets/galaxies will not be able to resist gravitation. They start to merge. The more merge they have, the greater the aged galaxies mass become. Those mega merged mass will crash matters within, feed black matters. Sooner or later, the inactive black hole/or matters will become active black holes due to ever increasing mass/energy within. The process of mega merge will become more and more dramatic or violent when it approaches to the boiling point ready for another big bang.

\subsection{Universe and its Cycle}

Based on observation, the universe is cooling. The peripheral galaxies are speeding up. Since the energy in the universe is constant, when the background temperature drops, the heat energy transforms into a different form of energy -that is momentum of aged galaxies. The increase of momentum of aged galaxies manifests as acceleration of peripheral galaxies. As a matter of fact, both heat and momentum belong to kinetic energy. The next question is: Why will the temperature drop? Based on the characteristics of all matter/materials, they tend to merge together in random movements- gravitation (a form of potential energy). In space, the galaxies are the most significant masses that gather in random movements when their speed drops. Their kinetic energy cannot offset inter-mass attractions or gravities. Beside of galaxies merging, the travel in universe space via vast distance, the traveling galaxies will inevitable pick up particles/cosmic strings/ray from background space because of gravity. The mega aggregation of galaxies/matter provides them ever-increasing heavier mass. More mass means more energy, in which some energy manifests in increasing momentum. Some of that energy will be used to condense the matter within-transforming to potential energy, which creates a heavier black hole. A heavier black hole simply means more energy or mass within. The heavier black hole (higher level of potential energy) will turn an inactive black hole to an active black hole (highest level of potential energy). The active black holes will absorb more mass. In the meantime, there are less matter/particles left in the background space of the universe after aging galaxies passing by, which not surprisingly, we will see a drop in temperature (losing matter means losing energy). Again, it is based on Einstein's equation -- the matter is energy. Energy, mass, and speed represent three fundamental characteristics of the matter/objects. They are not three separate things. If there is no energy, there will be no speed, and no mass. Similarly, if there is no mass, then no speed and no energy will exist. And if there is no speed, there will be no mass and no energy. Therefore, an absolute temperature of $0 \mathrm{~K}$ for any space means no movements, but it also tells us that no mass/energy exists in such space-a total void vacuum. Again, lost matter in the background means lost energy, which means a drop in temperature. Also based on the same characteristics of matter/materials conducted in random movements, gigantic numbers of regional galaxies/mass will eventually join together and reach a critical mass level or a critical energy level that can be labeled as a blast point/nuclear boiling point, then, it will trigger a gigantic explosion, similar to our nuclear reaction. The gigantic blast is also known as a big bang that simply bombs everything within to its most primitive state. The big blast spits out those most primitive particles, which one day will again form atoms, then molecules -- first inorganic, then organic. The organics will eventually become "alive" when they evolve to the point that they own boundaries and are capable of reproducing. As a matter of fact, the living organism will install orders in random world. For example, the tree turns dirt into a highly organized material form - e.g. tree trunk, barks and leaves. Not surprisingly, organism appreciates everything that is in order. The top organisms/humans appreciate beauty by our nature design as well. What is beauty? The beauty means organized/balanced objects. The higher level of civilization means superior in organizing. A chaotic nation or its army is a weak nation/army. In a competitive world, the more organized civilization will defeat or restore orders in less civilized or chaotic nations/regions. This is designed by nature. It is not a moral call. As we witness in our human history, many civilization have been wiped out by advanced ones. In the universe, some particles may escape the fate of being dragged into molecular structures. Those particles disperse and flow in universe space freely; thus, provide the background temperature in the universe. Some people call them cosmic strings/rays. They are the free-standing loners in the universe. What is gravity? The characteristics of matter tend to gather together in random movements, also known as gravitation (a form of potential energy). When matter/particles reach certain quantities in mass, that mass will become very noticeable (increasing potential energy). The only reason that masses such as planets/moons do not merge is because they revolve with a certain level of speed (kinetic energy) that temporarily offsets the gravity (gravitation potential) - the delicate balance between momentum/kinetic energy and gravitation/potential energy; but eventually they will merge, due to decline of the kinetic energy, which means evolving speed drops as time elapses. Gigantic merged mass will collapse within and crash matters to its primitive state-sub atom particles. The universe is nothing but an unending cycle of merging and collapsing first, which triggers a gigantic explosion eventually when the mass/energy reaches a critical blast point. The blast then spits out primitive particles that once again form molecules first, and then form galaxies/planets. The mother blast also fuels the mass with initial momentum/kinetic energy. When the kinetic/momentum energy declines to certain levels, those galaxies cannot resist the attractions of gravity. They once again merge and collapse, then trigger another explosion; then a new collection of galaxies will be formed, then they age, then collapse and merge again. It is hard to observe an exact chain reaction of events from point A to point B - for example, turning a big bag of dirt into a pink pig. However, I can assure you how easy it is to bomb a pink pig back into a bag of dirt. If point $\mathrm{A}$ to point $\mathrm{B}$ is constructive, then the 
opposite direction is destructive. Every construction is destined to destruction. On the other hand, every destruction also breeds new construction. It is like the yin and yang of the universe. The micro level of yin and yang of this universe cycle is that the yin characteristics of gathering features of matter/particles in random movement (potential energy), and the yang character of explosion when energy/matter reaches its blast point (kinetic energy). Yin represents Potential Energy, Yang represents Kinetic Energy. The entire universe is nothing but a cycle of energy transforming, from "yin" Potential Energy predominant transform to "yang" Kinetic Energy predominant, then back to "yin" Potential Energy predominant again-an endless cycle of energy transforming with no beginning or ending.

\subsection{Time, Death and Sleep}

Time is just a record of history that describes an evolution in process or movement in matter; if you were to stop time, you would see a snapshot or momentary freeze picture of matter physics -- a halted progress in matter/energy. The speed of matter/mass or their evolution can never stop; therefore, time will never stop. There is no disappearance of matter/mass/energy; there is only transformation of the engaging or dissembling with external or internal mass/energy. The so-called death in living organisms is really just a halt in it's thinking/emotion process. The material of death body will be recycled every bit in nature. The capital punishment is just stopping that particular thinking process/behavioral pattern; the material body of criminal cannot be erased. In terms of stopping the thinking process, sleep most closely resembles death. The difference is timing. Death means to stop the train of thinking for good. In near future, our medicine will rewrite the definition of human life. There will be two form of living being. One form is traditional human, for example, a functional physical body with all memories associated with his/her growth. Another form is electronic form, a person's memory and it's associated emotion which store in his/her brain as electronic data, that can be upload, transfer, then down load in computer hard drive or another functional physical alive body. In a way, the patient is diseased, because his/her original body is gone or decayed, but his/her memories are all uploaded and downloaded into a computer or another physical body, he/she is not really dead per se, because his/her memories and associated emotions with abilities unique to him/her can be aroused or "switch on", therefore continue to communicate with people. The process will be similar to transferring a song from old disc to a new disc. The song is still same song, but carrier is totally different. Death, as we define it today, will have to be re-written. The medical advancement will eventually affect space exploration as well. In future, we can send physical form of humans/astronauts to a distant planet-the traditional way, or we can send electronic form of humans to a distant planet as long as it has a lab established ahead of time where it can receive signals from earth, once the alien planet lab is capable to down load those signals (electronic form of astronauts) into their computer hardware. The electronic form of humans will be able to perform any duties that physical forms of humans are capable. The human space travel will potentially reach the speed of light.

\subsection{Mathematic Odds and Déjà Vu}

Since universe matters are all made of same particles, whether they are gods particles or ghost particles, there is no mystery or magic force in our universe. It is all about odds in the universe where math comes to play. From the most primitive particles to the most sophisticated chemical structures, matter will never vanish. It just exists in different forms by pure odds. The so-called "odds" just describe the chance of matter entering into a particular state. $\mathrm{E}=\mathrm{MC}^{2}$. Energy, mass, and speed: these three characteristics describe all materials/matter/particles/cosmic strings or you name it, regardless of its stage in matter evolution. Matter at different speeds not only means different levels of energy within, but also manifests different physical characters. The law applies one stage of matter may not apply the other stage. The neverending of cycle of evolution in matter/particles happens throughout the universe. Given the infinite size of the universe, the probabilities are most likely infinite as well: "anything is possible." Therefore, at any moment, if you take a snapshot, you can always find materials/particles of same physical state at different places in other parts of universe simultaneously, which gives people a sensation of déjà vu. Similarly, when you look into a large crowd, I am sure some déjà vu is going to play right in front of your eyes -- two faces will appear to be identical. Some scientists may refer it as a parallel universe or alternative universe. But there is no such thing. There is no parallel universe - if there were, it would violate the definition of "Universe." When you see two exactly identical coins, do you think that two coins are same one or parallel image? Another phenomenon occurs with the speed of light in vacuum. Some scientists feel that one light particle occurs in two different locations at the same time when they reach the speed of light. First of all, such observation is not valid, since no scientist is able to manipulate an environment to a true vacuum. However, I will not be surprise to know that at the speed of light, particles will demonstrate some unique physical character. Environment determines the physical state/character of particles/matter. However, the true void space/environment doesn't make matter/mass increase or decrease if no other matter/particles/energy exist which may collide into it or loses it to other matters along the way. If the same particle exists in two locations at the same time, that would mean double mass/energy during the pure speed of light in a vacuum (void space from any matter/energy). It means energy/mass can be doubled in the speed of light. If it were true, the energy cannot be constant in our universe. Such a statement simply violates the law that mass/matter will not increase if no foreign mass/matter/energy engages, or mass/matter will decrease if no energy/mass/matter is lost in the process. If mass/energy can be increased or doubled out of nothing in certain environments, then our universe's 
mass/energy will not be constant. When you see some matter/particles are at same stage of their respective evolution processes, but in different locations of the universe, you just need to remember that identical doesn't necessarily mean they are the same matters. They may give us an impression of mirror image to each other, but again they are not the same. The light particle some scientist saw in "their vacuum" appears to exist in two different locations simultaneously; they are actually two different particles. In a "not so pure" vacuum, one particle may very well attract another "foreign" particle pop up in its neighborhood due to its gravitation at micro level - a potential energy that a light particle possesses. In one word, there is no evidence that Einstein Equation is obsolete when it comes to particle world. Time should be simply explained as a history book, a record of chronicled events. Given the sheer number of possibilities in an infinite universe of both matters and space, many of the same events will occur in different parts of the universe. When you look into the universe, you might see the same events/matters of any form exist and progress in a different part of location of universe, the famous déjà vu. Finally, time is not made of particles; it does not follow the laws of physics. The time machine is another human fantasy.

\subsection{Physical Environment is the Determination Factor along with Odds}

Environment determines the physical state of matter/particles. How energy changes all depends on the environment where relevant matter/particles exist. The environment is an energy field filled with different interplaying matter/particles/mass. There is no place where is total void of energy/matter exists in any settings-natural or man-made. It is very questionable that we humans can ever create a space that is total void of any energy $(0 \mathrm{~K})$ - a completely void space must have a barrier that resists any particle penetration. It is a very bad news for particle physicists. The only solution may again require computer simulation. The ultimate space exploration will rely on computer simulation. Our entire universe is filled up with energy; it is everywhere. The only way to change the physical state of matter/particles/mass is through manipulating its related environment. Reciprocally, we can observe any state of matter/particles/mass by mimicking a different type of environment, then watch how matter/particles act in such environment. The infinite size of universe is formidable for any living organism to physically explore, not to mention the difficulty for any living being to actually evolve intelligently enough to escape the big bang. Future explorations of the universe, I believe, will have to rely on computer simulations. While we use computer to create various environmental situations, then we can see how matter/particles evolve or act in those environments. Hopefully, some of those cyber spaces can be true vacuum. The computer simulations may partially satisfy our insatiable appetite for knowing the unknown. In other words, computers will compensate for our inherited physical limitations.

\section{Results Analysis}

\subsection{Big Bangs}

During the life cycle of individual big bang, was it just a single big bang that created this current universe some 14 billion years ago? To say that, it will be like saying the entire world's population descended from a single mother. The world population is limited, but the universe is infinite. Let's see a nuclear reaction of current model, the level of blast/boiling point is quantified (not infinite), that means there is quantified matter/energy involved, which doesn't need infinite energy or matter engaged to trigger a nuclear reaction. Similarly, the big bang/particle explosion is triggered by a certain level of mass/energy. It cannot be an infinite level of mass/energy. It is another logical call! If an infinite level of mass and energy are required to trigger a big bang, how can a big bang ever happen? We would be stuck in a process of reaching an infinite level of mass/energy forever. I can safely say that infinite means "forever" here. If this were true, there would be no such thing as a big bang. There would be no start off and no end for any cycle of energy/mass evolution, because no such big bang would ever be triggered or happened. Other words, each bang may happen as long as a blast point is reached by collecting of measurable mass or galaxies/matter. Therefore, we can safely say that the universe is infinite, but the big bang is not. It only requires a limited quantity (enough to trigger a big blast) of mass/energy. The limited quantity, though gigantic in our eye, certainly cannot represent the entire universe (remember, the universe is infinite!); therefore, the entire universe (infinite) cannot be created by a single bang (finite). It is another logical call! At any given time, I can, therefore, safely reason that different parts of the universe are evolving at different stage. Some parts of the universe may be at newborn stage that just had a big blast; some parts of the universe are at a mature stage (e.g. ours); and some parts of the universe are old and dying, which will feed a new big bang. The mass engage in a new big bang may takes up matter/particles or cosmic strings from other parts of the universe while they travel in the space; therefore, the materials fed into a single big blast may not necessarily be from a single dying part of the universe. There are constant matter exchanges from different regions in the universe, because not all particles/matter will be trapped into molecular structures. Those free loners are the true free spirits in the universe; they may travel far away from their mother bang and enter territories of other mother bang's families. The fate of those particles/matters is to remain free until they are attracted to nearby passing galaxies/masses.

Those free particles will eventually participate in a big bang somewhere in the universe. That's also why some scientists feel like the matters engaged in this pre-blast bang of current "universe" can be traced to a very small therefore potentially measurable ball, and there are other "universes" existing parallel to our universe. Their six senses are correct in a way. Because each big bang only involves a limited amount of mass/matter/particles/energy (a measurable degree 
of boiling point). The matters/particles have their free loners that travel outside of their mother bang; those free loners may influence other parts of the universe and provide universe background temperature. Understandably, any new big bang may also involve dying galaxies from mass descended from different mother bang origins, because they might close enough to be caught in a new pre- big bang mass. To simplify the situation, we can generally state the universe is made of different parts(Zolaxys); each part (Zolaxy) has its own mother bang, though they are interweaved, especially the free mass/matter wander in different regions constantly.

\section{Discussion}

In this theoretical study, to put everything in a more organized way without greatly altering definition of universe, I will have to coin a new term for each part of the universal system - Zolaxy, which is created by a single mother blast. The Zolaxy is a family of galaxies created by a single big blast. In other words, the galaxies within any Zolaxy should have the same birth origin/mother bang. There may be different sizes and different shapes of Zolaxies, just like galaxies. Zolaxies are enormous in their size, and too far for us to see from our Zolaxy. Galaxies are much smaller and much closer, so we are able to observe them. So far, we have observed spiral galaxies (our Milky Way galaxy is a spiral galaxy), elliptical galaxies, and irregular galaxies*. If there are different types of galaxies, then most likely there are different types of Zolaxies. It all depends on the environment and energy/mass involved in that particular blast. Again, the Zolaxy can be categorized as a group of galaxies that have the same birth origin from a single mother bang. A Zolaxy is not the entire universe, just like the Milky Way is not an entire universe. As a matter of fact, our Zolaxy may represent a very tiny fraction of our infinite universe. The universe is infinite; the Zolaxy is limited in its size. The center of the Zolaxy is the origin site of the explosion; the newer galaxies are closer to the center of explosion. The aged galaxies are farther away from blast site. Understandably, travel cost energy; however, the aged galaxies are also picking up matter/particles/cosmic strings along the way. From a vast distance, the galaxies we observe are sped up, because those galaxies which have travelled vast distance are exposing, therefore picking up more matter/particles/mass from space. The ever increasing mass/galaxies shall manifest with greater momentum. When we watch the periphery galaxies, the momentum is picking up overall because they are fed by mass/matters in space which is indirectly proved by dropping universe's background temperature that is due to loss mass/matters to traveling galaxies. There is energy shift overall in universe secondary to travelling masses/galaxies. At the individual galaxy level, the longer distance Galaxies/Masses travel, the more matter /cosmic strings/energy are available to them. Their mass will grow heavier when they travel further and further (greater mass does not necessarily mean greater size), harvest the matter/particles from the background. Understandably, it will leave the background energy/matter scarce, though it will never vacuum clean the background space, leave background universe to its void of any energy/matters. Remember, there are always free particles/matters/mass (infinite numbers) wandering from place to place in universe. The bigger mass means more energy or greater momentum. When multiple aging galaxies merge and collapsed, this will feed the black hole to its higher level/active physical status; then more free standing matter/particles/energies will be harvested along the way until the gigantic collections of mass happens. At some point, the critical level of mass/energy is reached, and then the final big blast is triggered- a mother bang. Unfortunately, no big bang will ever be witnessed, because it is either too far away in distance or too long ago in time, or both. Computer simulations will be the only way for us to explore the all possibilities of interplay particles in universe.

\section{References}

[1] Paul Steinhardt of Princeton and Andreas Albrecht of University of California Davis. Scientists revise Big Bang theory: there may be multiple universes march 23. 2013.

[2] Dr Sebastien Muller of Onsala Space Observatory at Chalmers University of Technology in Sweden and..Nature's Most Precise Clocks May Make "Galactic GPS" Possible Francis Reddy Goddard Space Flight Center NASA's Fermi Gamma-ray Space Telescope is an astrophysics and particle physics partnership, developed in collaboration with the Department of Energy, along with important contributions from academic institutions and partners in France, Germany, Italy, Japan, Sweden, and the U.S.

[3] Edwin Zong Understanding of Universe, Time, Odds and Environment 2011 